\title{
Cul4b Promotes Progression of Malignant Cutaneous Melanoma Patients by Regulating CDKN2A
}

\author{
Chao Zhang, ${ }^{1}$ Can Cao, ${ }^{1}$ Xiu-li Liu, ${ }^{2}$ Tan $\mathrm{Jun}^{3}$ and Pei Liu ${ }^{4}$ \\ ${ }^{1}$ Department of Dermatology, The Second Affiliated Hospital of Shandong First Medical University, Taian, \\ Shandong, China \\ ${ }^{2}$ Department of Neonatal, Gaotang People's Hospital, Liaocheng, Shandong, China \\ ${ }^{3}$ Department of Dermatology, Shaanxi Provincial People's Hospital, Xi'an, Shaanxi, China \\ ${ }^{4}$ Department of Burn and Plastic Surgery, Qilu Hospital of Shandong University, Jinan, Shandong, China
}

\begin{abstract}
Although several molecular targeted therapy and immunotherapy have been developed, cutaneous melanoma prognosis is still not satisfying. Cul4b promotes the progression of several malignant tumors by regulating cell proliferation. However, its prognostic role in malignant cutaneous melanoma has not been evaluated. In this study, immunohistochemistry was performed to assess the expression of Cul4b in a consecutive patient cohort. The prognostic role of Cul $4 \mathrm{~b}$ was estimated with univariate and multivariate analysis. Cul4b was knocked down in melanoma cell line to evaluate its role in promoting cell proliferation. The results revealed that Cul4b was highly expressed in some of the cutaneous malignant melanoma patients and high expression of Cul4b was associated with poor melanoma-specific overall survival and poor disease-free survival. Cul4b expression was associated with Breslow categories, Clark level, and Ki67 expression. Univariate and multivariate analysis revealed that Cul4b is an independent prognosis risk factor of cutaneous melanoma. Downregulation of Cul4b inhibited the proliferation ability of melanoma cells and downregulated the expression of CDKN2A. These results suggest that Cul4b plays an essential role in cutaneous melanoma progression and may serve as a promising treatment target.
\end{abstract}

Keywords: CDKN2A; Cul4b; cutaneous melanoma; proliferation

Tohoku J. Exp. Med., 2021 May, 254 (1), 33-39.

\section{Introduction}

Melanoma is a malignant tumor deriving from melanocytes of any part of the body, including the skin and eyes, the mucosa of the genitals, and the anus and mouth (Shain and Bastian 2016, Tsao et al. 2017). The incidence rate of cutaneous melanoma has increased rapidly in the world (Abbas et al. 2014, Lugovic-Mihic et al. 2019, Rastrelli et al. 2014). While surgical resection is the critical treatment of melanoma, which provides a cure chance for patients without metastasis, most patients were found with metastasis at diagnosis and surgical resection only improves the survival time. New treatment regimes, such as biotherapy, immunotherapy, and targeted therapy [e.g., B-Raf protooncogene (BRAF) inhibitors], have been developed to treat patients with metastasis (Turner et al. 2018). The development of BRAF inhibitors has changed the clinical treatment of advanced melanoma and provided a more promising choice for advanced melanoma patients (Diaz-Martinez et al. 2018, Flaherty et al. 2010, Holderfield et al. 2014, Menzer et al. 2019). Despite the short duration of the response, patients treated with BRAF inhibitors obtained a high objective response rate (ORR) and often reached significant tumor regression effects. At present, in addition to BRAF targets, identification and development of more new gene targets is urgently needed.

Cell proliferation was a significant malignant cutaneous melanoma biological behavior which is associated with poor patient survival(Wei et al. 2019). Loss of function mutations of several pivotal targets in cell cycle regulation has been reported in cutaneous melanoma. Cul4b belonged to the E3 ubiquitin ligase family and was initially reported to be associated with $\mathrm{X}$-linked mental retardation syndrome (Badura-Stronka et al. 2010, Zhao and Sun 2012, Zou et al. 2007). Recent studies have revealed that $\mathrm{Cul} 4 \mathrm{~b}$ participates in various biological processes ( $\mathrm{Li}$ et al. 2020, Qi et al.

Received January 28, 2021; revised and accepted March 18, 2021. Published online May 20, 2021; doi: 10.1620/tjem.254.33.

Correspondence: Pei Liu, Department of Burn and Plastic Surgery, Qilu Hospital of Shandong University, 107 Wenhuaxi Road, Jinan, Shandong 250012, China.

e-mail: liupei_peiliu@126.com

(C)2021 Tohoku University Medical Press. This is an open-access article distributed under the terms of the Creative Commons Attribution-NonCommercial-NoDerivatives 4.0 International License (CC-BY-NC-ND 4.0). Anyone may download, reuse, copy, reprint, or distribute the article without modifications or adaptations for non-profit purposes if they cite the original authors and source properly.

https://creativecommons.org/licenses/by-nc-nd/4.0/ 
2019, Zou et al. 2013). As a E3 ubiquitin ligase family member, Cul4b regulates the ubiquitination of $\mathrm{H} 3 \mathrm{~K} 119$ and regulates downstream gene transcription ( $\mathrm{Hu}$ et al. 2012). $\mathrm{Cul} 4 \mathrm{~b}$ also regulates the expression of cyclin-dependent kinase 2 (CDK2) and Cyclin D1 by regulating miR-372 (Duan et al. 2020, Liu et al. 2020). However, as a significant cell cycle regulator, the prognostic role of $\mathrm{Cul} 4 \mathrm{~b}$ in the progression of cutaneous melanoma has not yet been studied.

In this study, the prognostic role of Cul4b in the progression of cutaneous melanoma has been evaluated in a consecutive patient cohort of our hospital. Univariate and multivariate analysis has been performed to evaluate the risk factors of our cohort. Downregulation of Cul4b was obtained in a melanoma cell line. Cell Counting Kit-8 (CCK8) and colony formation analysis was performed to examine the role of $\mathrm{Cul} 4 \mathrm{~b}$ on cell proliferation. Our results revealed an important role of $\mathrm{Cul} 4 \mathrm{~b}$ in the progression of cutaneous melanoma and indicates that Cul4b may be a promising treatment target.

\section{Material and Methods}

\section{Patients}

This study was approved by the Ethics Committee of The Second Affiliated Hospital of Shandong First Medical University (KYLL-2017-126). Written informed participation consents were obtained from all enrolled patients. Tumor tissues from 74 patients who underwent surgical resection in our hospital from January 2007 to December 2012 were obtained, formalin-fixed immediately after the resection of the tumor and then paraffin-embedded. The follow-up duration of all participants ranged from 16-120 months. The pathological type of the experimental samples used in this study were determined and confirmed by two senior pathologists of our center.

\section{Immunohistochemistry (IHC) staining}

IHC staining for Cul4B was performed according to the manufacturer's manual. Sections were first roasted at $70^{\circ} \mathrm{C}$ for $1 \mathrm{~h}$, dewaxed, deparaffinized by xylene and then rehydrated by alcohol gradients. Subsequently, sections undergone microwave antigen retrieval in $\mathrm{pH} 6.0$ citrate buffer. After that, the samples were blocked with normal goat serum, and incubated with the mouse anti-human Cul4B antibody (1:100 dilution; Cat. No. MABC556; Sigma-Aldrich, St. Louis, MO, USA) for at least $14 \mathrm{~h}$ at $4^{\circ} \mathrm{C}$. Finally, the specimens were incubated with HRPconjugated IgG and 3,3'-diaminobenzidine substrates to visualize the expression level. Expression of Cul4B in the tumor tissues was judged by two experienced pathologists. The H-score method was performed to quantify the expression of Cul4B in these tissues. The score was calculated according to the following formula: (\% cells of $1+$ intensity score $\times 1)+(\%$ cells of $2+$ intensity score $\times 2)+(\%$ cells of $3+$ intensity score $\times 3$ ). Patients were divided into high and low expression groups based on the median value of H-scores.

\section{Cell culture and transfection}

A375 were purchased from the Cell Bank of the Chinese Academy of Sciences (Shanghai, China), incubated at $37^{\circ} \mathrm{C}$ with $5 \% \mathrm{CO}_{2}$ and determined to be mycoplasmafree. Small hairpin RNAs (shRNAs) plasmids of Cul4b were purchased from Genomeditech Co., Ltd. (Shanghai, China). The transfected cells were screened with puromycin $(2 \mu \mathrm{g} / \mathrm{ml})$ for 1 week to establish stable cell lines. The target sequences of shRNA-1 and shRNA-2 were 5'-CAATCTCCTTGTTTCAGAA-3' and 5'-GAACTTCC GAGACAGACCT-3', respectively.

qRT-PCR

Total RNA was extracted using TRIzol reagent (Invitrogen; Carlsbad, CA, USA) and reverse-transcribed using the cDNA Synthesis kit (Takara; Kusatsu, Shiga, Japan). The RNA quantity and density were verified by a spectrophotometer. qRT-PCR was performed using the SYBR Green Master MIX Kit (Takara) according to the manufacturer's instructions. The assays were operated in triplicate and relative gene expression was determined by using the $2-\Delta \Delta \mathrm{Ct}$ method. The following primers were used: Cul4B-Forward: 5'- ACTCCTCCTTTACAACCCAG G-3'; Cul4B-Reverse: 5'- TCTTCGCATCAAACCCTACA AAC-3'; GAPDH-Forward: 5'- TGTGGGCATCAATGGA TTTGG-3'; GAPDH-Reverse: 5'- ACACCATGTATTCCG GGTCAAT-3'.

\section{Western blot}

For total protein extraction, cell lysates were obtained using RIPA buffer (Beyotime, Shanghai, China) supplemented with phosphatase and protease inhibitors (Yeasen, Shanghai, China). A total of $15 \mu \mathrm{L}$ protein was injected into a Bis-Tris SDS/PAGE gel and transferred to PVDF membranes. After blocking with 5\% bovine serum albumin (BSA), the membranes were incubated with primary antibody overnight at $4{ }^{\circ} \mathrm{C}$. Membranes were then exposed to the secondary antibody for $60 \mathrm{~min}$. The bands were visualized with an ECL kit, and were analyzed with an imaging system.

\section{CCK8}

For CCK-8 assay, 1,000 cells were inoculated into each well of 96 -well plates. At each time point $(0,24,48$, 72 and 96 h), $10 \mu \mathrm{L}$ CCK-8 solution (Yeasen, Shanghai, China) was added into the wells containing $90 \mu \mathrm{L}$ culture medium. The wells were incubated for $2 \mathrm{~h}$, and the absorbance of each individual well was determined at $450 \mathrm{~nm}$. Obtained data are presented as a line chart.

\section{Colony formation}

For colony formation assay, cells were digested into single-cell suspension and 1,000 cells seeded in each culture dish $(6 \mathrm{~cm})$. The appropriate complete medium was 
added to each dish with the culture medium being refreshed every three days. The cells were then washed with phosphate-buffered saline (PBS), fixed with $4 \%$ paraformaldehyde and stained with $0.4 \%$ crystal violet for $15 \mathrm{~min}$. The number of colonies containing $>10$ cells was counted manually and averaged from the duplicate wells.

\section{Statistical analysis}

SPSS version 25.0 software was applied for statistical analysis. Graphpad Prism 6.0 was applied to draw the survival analysis plots. Chi-square analysis was performed to analyze the relationship between clinical characteristics and the Cul4B expression. Risk factors of cutaneous melanoma were evaluated with the univariate and multivariate analysis. Kaplan-Meier method was applied to draw the diseasefree survival and overall survival curves. $\mathrm{P}<0.05$ was considered statistically significant.

\section{Results}

High Cul4b expression in malignant cutaneous melanoma indicates poor patient prognosis

To evaluate the role of $\mathrm{Cul} 4 \mathrm{~b}$ in the progression of malignant cutaneous melanoma, $\mathrm{Cul} 4 \mathrm{~b}$ expression was first evaluated in 74 primary tumors. Cul4b was expressed in various fractions and intensities as demonstrated by the typical picture of the high and low Cul4b expression in Fig. $1 \mathrm{~A}, \mathrm{~B}$. Cul4b expression was then evaluated by combining analysis of $\mathrm{Cul} 4 \mathrm{~b}$ expression fractions and intensities as $\mathrm{H}$ score. Next, the prognostic value of Clu $4 \mathrm{~b}$ expression was examined in SPSS according to a dichotomized variable of low and high Cul4b H score. As demonstrated in Fig. 2, Kaplan-Meier analysis revealed a decreased disease-free survival (DFS) and melanoma-specific survival (MSS) with increasing $\mathrm{H}$ score of $\mathrm{Cul} 4 \mathrm{~b}$ expression (Fig. 2A, B). Associations between low and high Cul4b expression, established prognostic factors and Ki67 expression are shown in Table 1. High Cul4b expression was significantly associated with unfavourable clinicopathological features, i.e., Breslow thickness $(p<0.001)$, Clark level $(p<0.001)$, and high Ki67 expression $(p=0.039)$.

The prognostic value of Clu $4 \mathrm{~b}$ and established prognostic factors for patient DFS and MSS was further evaluated in univariable and multivariable model Cox regression analysis (Table 2). Univariates analysis revealed that Breslow categories, vascular invasion, mitotic count and Ki67 expression and $\mathrm{Cul} 4 \mathrm{~b}$ expression were prognostic factors for patient DFS while Breslow categories, clinical stage, vascular invasion, mitotic count, Ki67 expression and
A

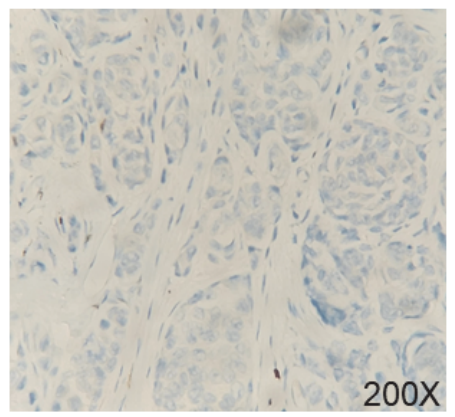

B

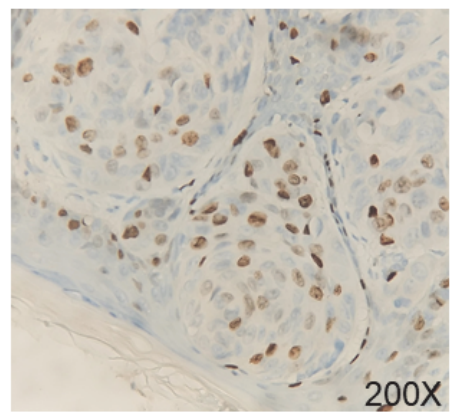

Fig. 1. Immunohistochemistry analysis of Cul4b expression in surgical resected tumor tissues of cutaneous melanoma. Cul4b expression differs from low (A) to relative high (B).

A



B

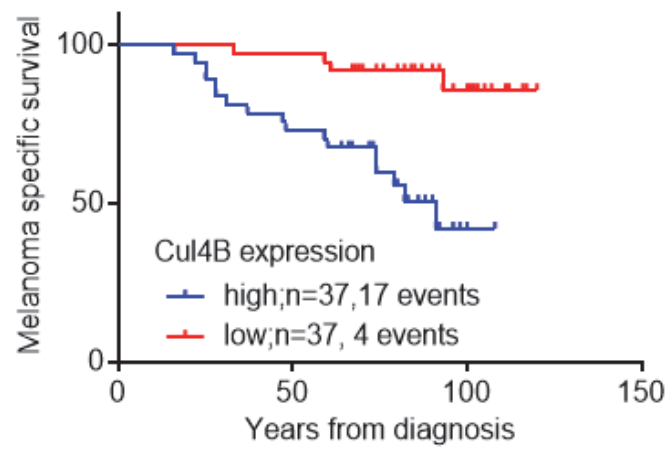

Fig. 2. Kaplan-Meier analysis of the effects of high and low Cul4b expression in surgical resected tumor tissues of cutaneous melanoma on patient prognosis.

High Cul4b expression indicates poor patient disease-free survival $(\mathrm{A}, \mathrm{p}=0.0003)$ and poor melanoma specific survival $(B, p=0.0002)$. 
Table 1. Relationship between intratumor Cul4B and clinicopathologic features.

\begin{tabular}{|c|c|c|c|c|}
\hline \multirow{2}{*}{ Variable } & & \multicolumn{3}{|c|}{ Intratumor Cul4B } \\
\hline & & $\begin{array}{l}\text { Low expression } \\
\quad(\mathrm{n}=37)\end{array}$ & $\begin{array}{l}\text { High expression } \\
\quad(\mathrm{n}=37)\end{array}$ & $\mathrm{p}$ \\
\hline \multirow[t]{2}{*}{ Age (years) } & $<50$ & 8 & 8 & 1.000 \\
\hline & $\geq 50$ & 29 & 29 & \\
\hline \multirow[t]{2}{*}{ Gender } & Male & 16 & 19 & 0.485 \\
\hline & Female & 21 & 18 & \\
\hline \multirow[t]{6}{*}{ Location } & perineum & 3 & 2 & 0.859 \\
\hline & legs & 13 & 11 & \\
\hline & Head and neck & 11 & 9 & \\
\hline & Dorsal and Frontal trunk & 3 & 3 & \\
\hline & Axillary and buttocks & 2 & 4 & \\
\hline & Arms & 5 & 8 & \\
\hline \multirow[t]{4}{*}{ Histological subtype } & Acral freckle & 15 & 19 & 0.689 \\
\hline & Malignant freckle & 12 & 8 & \\
\hline & Superficial spreading & 5 & 6 & \\
\hline & other & 5 & 4 & \\
\hline \multirow[t]{4}{*}{ Breslow categories } & $\leq 1 \mathrm{~mm}$ & 24 & 5 & 0.000 \\
\hline & $1.1-2.0 \mathrm{~mm}$ & 7 & 8 & \\
\hline & $2.1-4.0 \mathrm{~mm}$ & 5 & 18 & \\
\hline & $\geq 4.0 \mathrm{~mm}$ & 1 & 6 & \\
\hline \multirow[t]{4}{*}{ Clark level } & II & 21 & 3 & 0.000 \\
\hline & III & 13 & 18 & \\
\hline & IV-V & 1 & 16 & \\
\hline & others & 2 & 0 & \\
\hline \multirow[t]{3}{*}{ Clinical stage } & 1 & 29 & 29 & 1.000 \\
\hline & $2-4$ & 7 & 7 & \\
\hline & unknown & 1 & 1 & \\
\hline \multirow[t]{2}{*}{ Vascular invasion } & no & 36 & 35 & 0.556 \\
\hline & yes & 1 & 2 & \\
\hline \multirow[t]{2}{*}{ Mitotic count } & $0 / \mathrm{mm}^{2}$ & 20 & 19 & 0.816 \\
\hline & $\geq 1 / \mathrm{mm}^{2}$ & 17 & 18 & \\
\hline \multirow[t]{3}{*}{ Lymphocytic infiltration } & None-mild & 10 & 13 & 0.479 \\
\hline & Moderate-brisk & 26 & 24 & \\
\hline & unknown & 1 & 0 & \\
\hline \multirow[t]{3}{*}{ Ki67 expression } & low & 29 & 19 & 0.039 \\
\hline & high & 5 & 14 & \\
\hline & unknown & 3 & 4 & \\
\hline
\end{tabular}

Cul4b expression were prognostic factors for patient MSS. Multivariable analysis further confirmed the prognostic role of Cul4b for patient DFS (HR $=4.649,95 \%$ CI 1.604 13.474) and MSS (HR $=6.042,95 \%$ CI 1.657-22.031).

Downregulation of Cul4b expression in melanoma tumor cells inhibits cell proliferation ability

As Cul4b expression was correlated with tumor Ki67 expression, we hypothesized that $\mathrm{Cul} 4 \mathrm{~b}$ may promote malignant melanoma progression by promoting cell proliferation. We then examined this hypothesis by downregulating Cul4b in melanoma cell line. As shown in Fig. 3A, downregulation of Cul4b was successfully established in A375 cell line. CCK8 analysis revealed that downregulation of Cul4b expression inhibited cell proliferation (Fig. 3B). Further colony formation analysis revealed that downregulation of $\mathrm{Cul} 4 \mathrm{~b}$ inhibited melanoma cell colony formation. Taken together, these results revealed that $\mathrm{Cul} 4 \mathrm{~b}$ promotes malignant melanoma progression by promoting cell proliferation.

CDKN2A plays a significant role in the development and progression of cutaneous melanoma (Betti et al. 2016, Hayward et al. 2017). However, as a significant cell cycle regulator, whether expression of CDKN2A was regulated 
Table 2. Univariate and multivariate analysis of factors associated with disease-free survival and melanoma specific survival rates of cutaneous melanoma following surgical resection.

\begin{tabular}{|c|c|c|c|c|c|c|c|c|}
\hline \multirow{3}{*}{ Clinicopathological factors } & \multicolumn{4}{|c|}{ Disease-free survival } & \multicolumn{4}{|c|}{ Overall survival } \\
\hline & \multirow{2}{*}{ Univariate P } & \multicolumn{3}{|c|}{ Multivariate } & \multirow{2}{*}{ Univariate $\mathrm{P}$} & \multicolumn{3}{|c|}{ Multivariate } \\
\hline & & Hazard ratio & $95 \% \mathrm{CI}$ & $\mathrm{p}$ & & Hazard ratio & $95 \% \mathrm{CI}$ & $\mathrm{p}$ \\
\hline Age $<50$ vs. $\geq 50$ & 0.903 & & & & 0.494 & & & \\
\hline Gender female vs. male & 0.481 & & & & 0.200 & & & \\
\hline Location & 0.134 & & & & 0.094 & & & \\
\hline Histological subtype & 0.175 & & & & 0.179 & & & \\
\hline Breslow categories & 0.004 & 1.600 & $1.042-2.458$ & 0.032 & 0.001 & 1.818 & $1.122-2.944$ & 0.015 \\
\hline Clark level & 0.105 & & & & 0.172 & & & \\
\hline Clinical stage & 0.102 & & & & 0.027 & & & \\
\hline Vascular invasion & 0.001 & & & & 0.000 & & & \\
\hline Mitotic count & 0.004 & 5.870 & $2.323-14.836$ & 0.000 & 0.031 & 4.965 & $1.756-14.039$ & 0.003 \\
\hline Lymphocytic infiltration & 0.126 & & & & 0.424 & & & \\
\hline Ki67 expression & 0.000 & 2.305 & $1.316-4.037$ & 0.003 & 0.000 & 3.559 & $1.746-7.251$ & 0.000 \\
\hline Cul4B expression & 0.000 & 4.649 & $1.604-13.474$ & 0.005 & 0.000 & 6.042 & $1.657-22.031$ & 0.006 \\
\hline
\end{tabular}

A

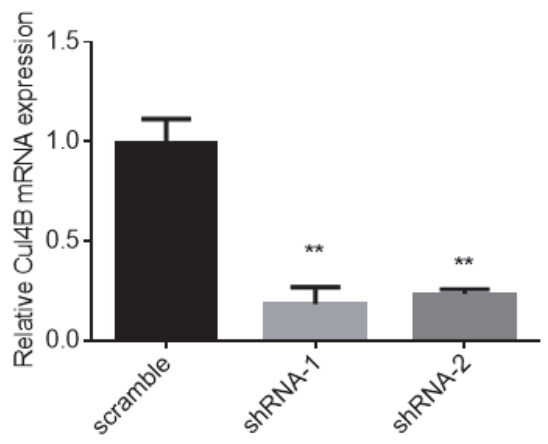

C

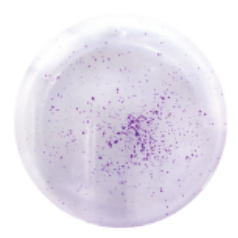

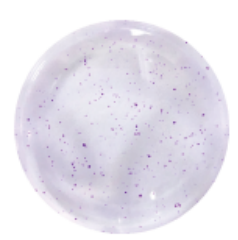

B
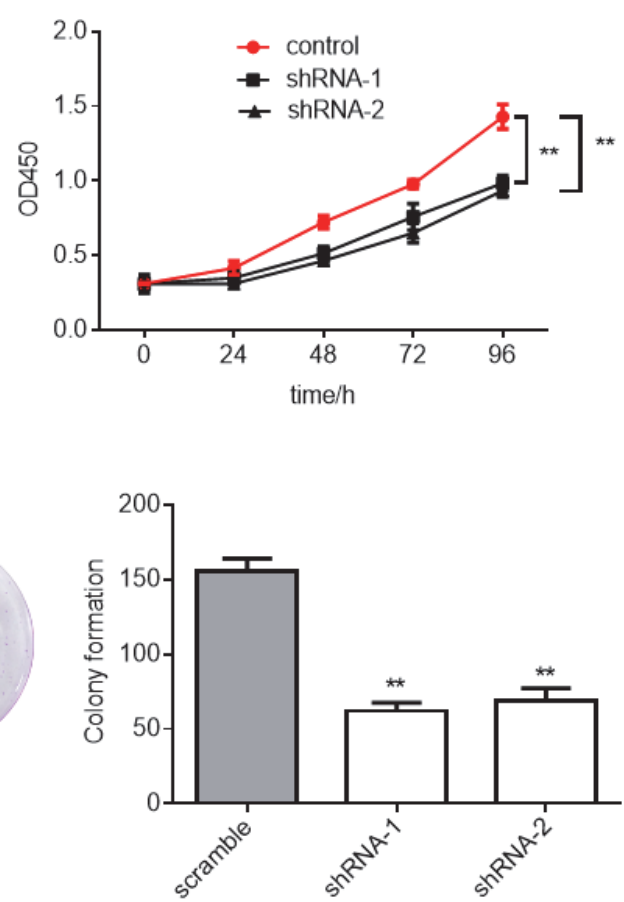

Fig. 3. Downregulation of Cul4b expression inhibited cell proliferation.

(A) Cul4b expression was downregulated in A375 cells. (B) CCK8 analysis of control cells and Cul4b knockdown cells. Cul4b knockdown cells were attenuated in cell proliferation ability. (C) Colony formation analysis of control cells and Cul4b knockdown cells. Less colonies were formed in Cul4b knockdown cells than control cells.

by Cul4b has not been evaluated. We then examined whether $\mathrm{Cul} 4 \mathrm{~b}$ promotes cutaneous melanoma progression by regulating $\mathrm{CDKN} 2 \mathrm{~A}$ in Cul4b knockdown cells. As shown in Fig. 4, knockdown of Cul4b results in downregulation of the expression of CDKN2A. These results indicate that $\mathrm{Cul} 4 \mathrm{~b}$ may promote cutaneous melanoma by upregulating the expression of CDKN2A.

\section{Discussion}

According to the global cancer statistics in 2018, it is estimated that there are more than 280, 000 new cutaneous melanoma cases in the world, accounting for $1.6 \%$ of the total new cancer cases and there are more than 60,000 death cases in the world, accounting for $0.6 \%$ of the total cancer 
CDKN2A

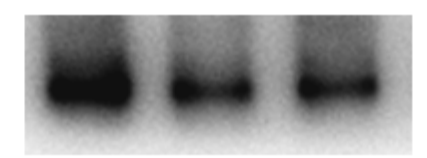

$16 \mathrm{kD}$

\section{GAPDH}

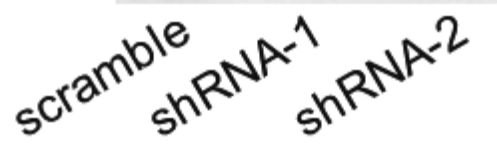

$37 \mathrm{kD}$

Fig. 4. CDKN2A was significantly downregulated in Cul4b knockdown cells.

deaths (Bray et al. 2018). The incidence rate and mortality rate of skin melanoma are relatively low in China (Wu et al. 2020). Although the incidence rate and mortality rate of melanoma are relatively low, the incidence rate is increasing at a rate of $6 \%-7 \%$ yearly. In the American Cancer Conference (ASCO) report, its incidence rate has undergone rapid increase (Abbas et al. 2014, Lugovic-Mihic et al. 2019, Mackiewicz and Mackiewicz 2012). Melanoma in Asian is quite different from that in Europe and the United States. Among Asians, about $50 \%$ of primary melanomas are in the extremities, and about $20 \%-30 \%$ are in the mucous membranes (Shen et al. 2019, Soft Tissue Tumor and Melanoma Group of Sarcoma Committee, Chinese Anti-Cancer Association 2020, Zhou et al. 2017). For Europe and the United States, about $90 \%$ of primary melanomas are in the skin and only $1 \%-5 \%$ of primary melanomas are in the mucous membranes.

DNA damage-induced uncontrolled cell proliferation may be the basic cause of melanoma. However, the exact cause of DNA damage in skin cells and the mechanism of melanoma are still unclear. To evaluate the histopathological and genetic prognostic factors of cutaneous melanoma, the pathological and clinical data of 74 patients with cutaneous melanoma in our hospital from 2007 to 2012 were retrospectively analyzed. Compared with previous studies, the sample size of this study is larger, taking advantage of the characteristics of China's large population base. The patients received the same treatment methods, which reduced the bias caused by different treatment modes. The results revealed that Breslow categories, mitotic count, Ki67 expression level as well as Cul4b expression level were significantly associated with the prognosis of cutaneous melanoma. Breslow categories, mitotic count and Ki67 expression level are common and well-known histopathological prognostic factors in cutaneous melanoma. Our results revealed that in Asian cutaneous melanoma patients, whose tumor mainly derive from extremities and mucosa, Breslow categories, mitotic count and Ki67 expression level are still the prognostic factors. Our results also for the first time revealed a prognostic role of Cul4b in Asian cutaneous melanoma.
We also explored the potential mechanism of Cul4b correlating with poor patient survival by downregulating the expression of $\mathrm{Cul} 4 \mathrm{~b}$ in melanoma cell lines. The results indicate that downregulating the expression of Cul4b inhibited melanoma cell proliferation and this may be due to the downregulated expression of cell cycle regulator CDKN2A. As Cul4b is reported to regulate cell cycle by regulating CDK2 and Cyclin D1, our study established a potential role of Cul4b in the regulation of Cyclin D1. However, as Cul4b regulates the expression of CDK2 and Cyclin D1 in a microRNA-dependent mechanism, it is worth further exploring whether $\mathrm{Cul} 4 \mathrm{~b}$ regulates the expression of CDKN2A in a microRNA-dependent mechanism or ubiquitination-dependent mechanism.

In conclusion, our results revealed that high $\mathrm{Cul} 4 \mathrm{~b}$ expression is correlated with poor patient disease-free survival and poor patient melanoma-free survival. Cul4b expression is associated with Breslow categories, Clark level as well as Ki67 expression level. Breslow categories, mitotic count, Ki67 expression and Cul4b expression level are independent prognostic factor of patient prognosis. Cul4b may promote melanoma cell proliferation by downregulating the expression level of CDKN2A. These results established a role of $\mathrm{Cul} 4 \mathrm{~b}$ in the progression of cutaneous melanoma and indicated that $\mathrm{Cul} 4 \mathrm{~b}$ may be a promising treatment target.

\section{Conflict of Interest}

The authors declare no conflict of interest.

\section{References}

Abbas, O., Miller, D.D. \& Bhawan, J. (2014) Cutaneous malignant melanoma: update on diagnostic and prognostic biomarkers. Am. J. Dermatopathol., 36, 363-379.

Badura-Stronka, M., Jamsheer, A., Materna-Kiryluk, A., Sowinska, A., Kiryluk, K., Budny, B. \& Latos-Bielenska, A. (2010) A novel nonsense mutation in CUL4B gene in three brothers with X-linked mental retardation syndrome. Clin. Genet., 77, 141-144.

Betti, M., Aspesi, A., Biasi, A., Casalone, E., Ferrante, D., Ogliara, P., Gironi, L.C., Giorgione, R., Farinelli, P., Grosso, F., Libener, R., Rosato, S., Turchetti, D., Maffe, A., Casadio, C., et al. (2016) CDKN2A and BAP1 germline mutations predispose to melanoma and mesothelioma. Cancer Lett., 378, 120-130.

Bray, F., Ferlay, J., Soerjomataram, I., Siegel, R.L., Torre, L.A. \& Jemal, A. (2018) Global cancer statistics 2018: GLOBOCAN estimates of incidence and mortality worldwide for 36 cancers in 185 countries. CA Cancer J. Clin., 68, 394-424.

Diaz-Martinez, M., Benito-Jardon, L., Alonso, L., Koetz-Ploch, L., Hernando, E. \& Teixido, J. (2018) miR-204-5p and miR211-5p contribute to BRAF inhibitor resistance in melanoma. Cancer Res., 78, 1017-1030.

Duan, P.J., Zhao, J.H. \& Xie, L.L. (2020) Cul4B promotes the progression of ovarian cancer by upregulating the expression of CDK2 and CyclinD1. J. Ovarian Res., 13, 76.

Flaherty, K.T., Puzanov, I., Kim, K.B., Ribas, A., McArthur, G.A., Sosman, J.A., O’Dwyer, P.J., Lee, R.J., Grippo, J.F., Nolop, K. \& Chapman, P.B. (2010) Inhibition of mutated, activated BRAF in metastatic melanoma. N. Engl. J. Med., 363, 809-819. 
Hayward, N.K., Wilmott, J.S., Waddell, N., Johansson, P.A., Field, M.A., Nones, K., Patch, A.M., Kakavand, H., Alexandrov, L.B., Burke, H., Jakrot, V., Kazakoff, S., Holmes, O., Leonard, C., Sabarinathan, R., et al. (2017) Whole-genome landscapes of major melanoma subtypes. Nature, 545, 175-180.

Holderfield, M., Deuker, M.M., McCormick, F. \& McMahon, M. (2014) Targeting RAF kinases for cancer therapy: BRAFmutated melanoma and beyond. Nat. Rev. Cancer, 14, 455-467.

Hu, H., Yang, Y., Ji, Q., Zhao, W., Jiang, B., Liu, R., Yuan, J., Liu, Q., Li, X., Zou, Y., Shao, C., Shang, Y., Wang, Y. \& Gong, Y. (2012) CRL4B catalyzes H2AK119 monoubiquitination and coordinates with PRC2 to promote tumorigenesis. Cancer Cell, 22, 781-795.

Li, Y., Hu, H., Wang, Y., Fan, Y., Yang, Y., Guo, B., Xie, X., Lian, J., Jiang, B., Han, B., Wang, Y., Shao, C. \& Gong, Y. (2020) CUL4B contributes to cancer stemness by repressing tumor suppressor miR34a in colorectal cancer. Oncogenesis, 9, 20.

Liu, X., Cui, J., Gong, L., Tian, F., Shen, Y., Chen, L., Wang, Y., Xia, Y., Liu, L., Ye, X., Wang, M., Liu, G., Jiang, B., Shao, C., Zou, Y., et al. (2020) The CUL4B-miR-372/373-PIK3CA$\mathrm{AKT}$ axis regulates metastasis in bladder cancer. Oncogene, 39, 3588-3603.

Lugovic-Mihic, L., Cesic, D., Vukovic, P., Novak Bilic, G., Situm, M. \& Spoljar, S. (2019) Melanoma development: current knowledge on melanoma pathogenesis. Acta Dermatovenerol. Croat., 27, 163-168.

Mackiewicz, J. \& Mackiewicz, A. (2012) Recent advances in melanoma treatment - American Society of Clinical Oncology (ASCO) 2012 perspective. Contemp. Oncol. (Pozn), 16, 197-200.

Menzer, C., Menzies, A.M., Carlino, M.S., Reijers, I., Groen, E.J., Eigentler, T., de Groot, J.W.B., van der Veldt, A.A.M., Johnson, D.B., Meiss, F., Schlaak, M., Schilling, B., Westgeest, H.M., Gutzmer, R., Pfohler, C., et al. (2019) Targeted therapy in advanced melanoma with rare BRAF mutations. $J$. Clin. Oncol., 37, 3142-3151.

Qi, M., Hu, J., Cui, Y., Jiao, M., Feng, T., Li, X., Pang, Y., Chen, X., Qin, R., Su, P., Zhang, H., Wang, Y., Gong, Y. \& Han, B. (2019) CUL4B promotes prostate cancer progression by forming positive feedback loop with SOX4. Oncogenesis, 8 , 23.

Rastrelli, M., Tropea, S., Rossi, C.R. \& Alaibac, M. (2014) Mela- noma: epidemiology, risk factors, pathogenesis, diagnosis and classification. In Vivo, 28, 1005-1011.

Shain, A.H. \& Bastian, B.C. (2016) From melanocytes to melanomas. Nat. Rev. Cancer, 16, 345-358.

Shen, X., Yu, R.X., Shen, C.B., Li, C.X., Jing, Y., Zheng, Y.J., Wang, Z.Y., Xue, K., Xu, F., Yu, J.B., Meng, R.S. \& Cui, Y. (2019) Dermoscopy in China: current status and future prospective. Chin. Med. J. (Engl.), 132, 2096-2104.

Soft Tissue Tumor; Melanoma Group of Sarcoma Committee, Chinese Anti-Cancer Association (2020) Chinese expert consensus on the surgical treatment of cutaneous/acral melanoma V1.0. Zhonghua Zhong Liu Za Zhi, 42, 81-93.

Tsao, H., Fukunaga-Kalabis, M. \& Herlyn, M. (2017) Recent advances in melanoma and melanocyte biology. J. Invest. Dermatol., 137, 557-560.

Turner, N., Ware, O. \& Bosenberg, M. (2018) Genetics of metastasis: melanoma and other cancers. Clin. Exp. Metastasis, 35, 379-391.

Wei, C.Y., Zhu, M.X., Lu, N.H., Peng, R., Yang, X., Zhang, P.F., Wang, L. \& Gu, J.Y. (2019) Bioinformatics-based analysis reveals elevated MFSD12 as a key promoter of cell proliferation and a potential therapeutic target in melanoma. Oncogene, 38, 1876-1891.

Wu, Y., Wang, Y., Wang, L., Yin, P., Lin, Y. \& Zhou, M. (2020) Burden of melanoma in China, 1990-2017: findings from the 2017 global burden of disease study. Int. J. Cancer, 147, 692-701.

Zhao, Y. \& Sun, Y. (2012) CUL4B ubiquitin ligase in mouse development: a model for human X-linked mental retardation syndrome? Cell Res., 22, 1224-1226.

Zhou, C., Wang, Y., Jia, R. \& Fan, X. (2017) Conjunctival melanoma in chinese patients: local recurrence, metastasis, mortality, and comparisons with caucasian patients. Invest. Ophthalmol. Vis. Sci., 58, 5452-5459.

Zou, Y., Liu, Q., Chen, B., Zhang, X., Guo, C., Zhou, H., Li, J., Gao, G., Guo, Y., Yan, C., Wei, J., Shao, C. \& Gong, Y. (2007) Mutation in CUL4B, which encodes a member of cullin-RING ubiquitin ligase complex, causes $\mathrm{X}$-linked mental retardation. Am. J. Hum. Genet., 80, 561-566.

Zou, Y., Mi, J., Wang, W., Lu, J., Zhao, W., Liu, Z., Hu, H., Yang, Y., Gao, X., Jiang, B., Shao, C. \& Gong, Y. (2013) CUL4B promotes replication licensing by up-regulating the CDK2CDC6 cascade. J. Cell Biol., 200, 743-756. 